\title{
Spatial Tail Dependence and Survival Stability in a Class of Archimedean Copulas
}

\author{
Diakarya Barro, ${ }^{1}$ Moumouni Diallo, ${ }^{2}$ and Remi Guillaume Bagré ${ }^{3}$ \\ ${ }^{1}$ UFR-SEG, Université Ouaga II, 12 BP 417, Ouagadougou 12, Burkina Faso \\ ${ }^{2}$ FSEG, Université des SSG, BP 2575, Bamako, Mali \\ ${ }^{3}$ UFR-ST, Université de Koudougou, BP 376, Koudougou, Burkina Faso
}

Correspondence should be addressed to Diakarya Barro; dbarro2@gmail.com

Received 27 February 2016; Accepted 17 May 2016

Academic Editor: Radko Mesiar

Copyright (C) 2016 Diakarya Barro et al. This is an open access article distributed under the Creative Commons Attribution License, which permits unrestricted use, distribution, and reproduction in any medium, provided the original work is properly cited.

\begin{abstract}
This paper investigates properties of extensions of tail dependence of Archimax copulas to high dimensional analysis in a spatialized framework. Specifically, we propose a characterization of bivariate margins of spatial Archimax processes while spatial multivariate upper and lower tail dependence coefficients are modeled, respectively, for Archimedean copulas and Archimax ones. A property of stability is given using convex transformations of survival copulas in a spatialized Archimedean family.
\end{abstract}

\section{Introduction}

In stochastic multivariate modeling, the use of copulas provides a powerful method of analysing the dependence structure of two or more random variables. Copulas were first introduced via a pioneering result of Abe Sklar (see Durante and Sempi [1]) by means of a theorem which bears his name and which constitutes the fundamental tool of copulas applications to dependence modeling in statistics. Since then, the copula functions appear implicitly in any multivariate distributions as a structure that allows separating the marginal distributions and the dependence model. Specifically, the $n$-dimensional copula $C$ associated with a random vector $\left(X_{1}, \ldots, X_{n}\right)$ with cumulative distribution $F=$ $\left(F_{1}, \ldots, F_{n}\right)$ is given by the probability integral transformation mapping $\mathbb{R}^{n}$ to $[0,1]$ via the Sklar representation (see [2]); such that, for all $\left(x_{1}, \ldots, x_{n}\right) \in \mathbb{R}^{n}$,

$$
F\left(x_{1}, \ldots, x_{n}\right)=C\left(F_{1}\left(x_{1}\right), \ldots, F_{n}\left(x_{n}\right)\right)
$$

Several approaches of construction of multivariate copulas have been developed and many surveys of copulas theory and applications have appeared in the literature to date. For instance, Joe [2] and Nelsen [3] are two key textbooks on copulas analysis, providing clear and detailed introductions to copulas and dependence modeling with an emphasis on statistical and mathematical foundations in spatial context.

Arising naturally in the context of Laplace Transforms (see Joe [2]), Archimedean copulas form a prominent class of copulas which leads to the construction of multivariate distributions involving one-dimensional generator functions (Charpentier and Segers [4]). Particularly, an $n$-dimensional copula $C$ is an Archimedean copula, if there exists a continuous and strictly decreasing convex function $\phi$, the generator of $C$, in the class of completely monotone functions:

$$
\begin{aligned}
& \{\phi:[0,+\infty] \longrightarrow[0,1] ; \phi(0)=1 ; \phi(\infty) \\
& \left.\quad=0 ; \quad(-1)^{k} \frac{\partial^{k} \phi^{-1}(t)}{\partial t^{k}} \geq 0 ; k \in \mathbb{N}\right\},
\end{aligned}
$$

with generalized inverse $\phi^{-1}(y)=\inf \{t \in[0,1], \phi(t) \leq y\}$ such that

$$
\begin{aligned}
C\left(u_{1}, \ldots, u_{n}\right)=\phi^{-1}\left[\phi\left(u_{1}\right)+\right. & \left.\cdots+\phi\left(u_{n}\right)\right] ; \\
& \forall\left(u_{1}, \ldots, u_{n}\right) \in[0,1]^{n} .
\end{aligned}
$$

Standard properties of Archimedean copulas can be found in McNeil and Nešlehová [5]. Particularly Nelsen ([6] or 
[3]) provides a list of common one-parameter Archimedean generator functions while Whelan [7] and Hofert and Scherer [8] studied algorithms for sampling Archimedean copulas. The class of Archimedean copulas started a wide range of applications for a number of properties like their computational tractability and their flexibility to model dependences between random variables. One of the most salient properties is the exchangeability induced by the algebraic associativity; that is, in bivariate case,

$$
\begin{aligned}
C\left(u_{1}, C\left(u_{2}, u_{3}\right)\right)=C\left(C\left(u_{1}, u_{2}\right), u_{3}\right) & \\
& \forall\left(u_{1}, u_{2}, u_{3}\right) \in[0,1]^{3} .
\end{aligned}
$$

However, for many other applications like risk managment, the exchangeability turns out to be restrictive. To overcome this drawback researchers have developed other approaches of modeling. For example, Joe [2] and Hofert and Scherer [8] use Laplace Transforms (LT) to derive more asymmetric and flexible extensions of multivariate Archimedean copulas. In the same vein and with the aim to construct Archimedean copulas belonging to given domain of attraction, Charpentier et al. (see [9]) introduced, in bivariate context, the family of Archimax copulas by combining the extreme values and Archimedean copulas classes into a single class. A member of this class with generator $\phi$ has the following representation:

$$
\begin{array}{r}
C_{\phi, A}(u, v)=\phi^{-1}\left\{(\phi(u)+\phi(v)) A\left(\frac{\phi(u)}{\phi(u)+\phi(v)}\right)\right\}, \\
(u, v) \in[0,1]^{2},
\end{array}
$$

where $\phi$ satisfies relation (1) and $A$ is a convex dependence function mapping $[0,1]$ to $[1 / 2,1]$ and verifying $\max (t ; 1-$ $t) \leq A(t) \leq 1$.

Particularly, in stationary geostatistical context, we have

$$
P(X<x, Y<y)=C_{\phi, A_{h}}\left(\phi^{-1}(x), \phi^{-1}(y)\right),
$$

where $h=|x-y|$ is the distance between the two sites.

The main contribution of this paper is to model multivariate tail dependence by extending this asymptotic result to spatial framework. Property of spatial stability of survival Archimax copulas is also proved. The paper is organized as follows: Section 2 gives the basic definitions and properties that will be needed for our results. Section 3 presents our main results. Thus, the parametric tail dependence is also generalized. Specifically, the upper parameter is analytically modeled via the LT-generator of the process and the lower parameter is characterized both for strictly Archimedean and for strictly extremal subclasses in parametric context.

\section{Materials and Methods}

In this section we collect the important definitions and properties on multivariate tail dependence and survival Archimax copulas that turn out to be necessary for our approach. The reader which is interested in bivariate statements of Archimedean copulas is referred to Genest and MacKay [10] and the software R and Evanesce [11] implements Archimax models proposed by Joe [2].
2.1. Multivariate Tail Dependence Coefficient. The concept of tail dependence of two random variables is related to the magnitude of the occurrence that one component is extremely large assuming that the other component is also extremely large. For two random variables $X$ and $Y$ with joint distribution function $F_{X, Y}=\left(F_{X}, F_{Y}\right)$, the upper tail dependence coefficient (TDC) is the conditional probability $\lambda_{U}$ (if it exists) that $Y$ is greater than the $100 \alpha$ th percentile of $F_{Y}$ given that $X$ is greater than the $100 \alpha$ th percentile of $F_{X}$ as $\alpha$ approaches 1; that is,

$$
\lambda_{U}=\lim _{\alpha \rightarrow 1^{-}} P\left(Y>\frac{F_{Y}^{-1}(\alpha)}{X}>F_{X}^{-1}(\alpha)\right) .
$$

Similarly, the lower TDC is defined, if it exists, by

$$
\lambda_{L}=\lim _{v \rightarrow 0^{+}} P\left(Y<\frac{F_{Y}^{-1}(v)}{X}<F_{X}^{-1}(v)\right) .
$$

Several approaches of generalization of TDC to high dimensional cases have been proposed.

Definition 1 (see Diakarya [12] or [13]). A multivariate generalization of the TDC consists in considering $k(k<n)$ variables and to quantify the conditional probability that each of the $k$ variables takes values from the tail given that the remaining $n-k$ variables take values from the tail too. For a given $k$, the corresponding generalized upper TDC is given by

$$
\begin{aligned}
\lambda^{U, k} & =\lim _{u \rightarrow 1^{-}} P\left(X_{1}>F_{1}^{-1}(u) ; \ldots ; X_{k}>F_{k}^{-1}(u) \mid X_{k+1}\right. \\
& \left.>F_{k+1}^{-1}(u) ; \ldots ; X_{n}>F_{n}^{-1}(u)\right) .
\end{aligned}
$$

Similarly, the lower TDC is given by

$$
\begin{aligned}
\lambda_{L, k} & =\lim _{u \rightarrow 0^{+}} P\left(X_{1} \leq F_{1}^{-1}(u) ; \ldots ; X_{k} \leq F_{k}^{-1}(u) \mid X_{k+1}\right. \\
& \left.\leq F_{k+1}^{-1}(u) ; \ldots ; X_{n} \leq F_{n}^{-1}(u)\right) .
\end{aligned}
$$

2.2. Radial Symmetry of Survival Distributions Functions. In multivariate modeling with Archimedean copulas in actuarial fields or duration analysis in economics, it is more appropriate to use the stochastic behaviours by means of survival function $\bar{F}$ of the distribution $F$ associated with the underlying copula.

Definition 2 (Cherubini et al. [14]). The survival function of the cumulative distribution function $F$ associated with a random vector denoted as $\bar{F}$ represents, if evaluated at $x=\left(x_{1}, \ldots, x_{n}\right)$, the joint probability that $X$ is greater than $x_{1}, \ldots, x_{n}$, respectively,

$$
\begin{aligned}
\bar{F}\left(x_{1}, \ldots, x_{n}\right) & =P\left[X_{1}>x_{1}, \ldots, X_{n}>x_{n}\right] \\
& =1+\sum_{K \in \nabla}(-1)^{|K|} F_{K}\left(x_{K}, K \in \nabla\right),
\end{aligned}
$$

where $\nabla$ is the set of nonempty subsets of $\{1,2, \ldots, n\}$ while $\left\{F_{K}, K \in \nabla\right\}$ denotes the set of lower marginal distributions of $F$. 
Thus, we derive from relation (11) the survival version of Sklar's representation (1) as

$$
\begin{aligned}
\bar{C}\left(u_{1}, \ldots, u_{n}\right)=F\left[\left(\bar{F}_{1}^{-1}\left(u_{1}\right), \ldots, \bar{F}_{n}^{-1}\left(u_{n}\right)\right],\right. \\
\forall\left(u_{1}, \ldots, u_{n}\right) \in[0,1]^{n},
\end{aligned}
$$

where $\bar{F}_{i}^{-1}$ are the generalized pseudoinverses of $\bar{F}_{i}$ (see Joe [2]).

Note that, in stochastic analysis, care needs to be taken when dealing with survival concept in multivariate case. Indeed, it follows that, for $n \geq 2$,

$$
\begin{aligned}
& \bar{F}\left(x_{1}, \ldots, x_{n}\right)=P\left(X_{1}>x_{1}, \ldots, X_{n}>x_{n}\right) \\
& \neq 1-F\left(x_{1}, \ldots, x_{n}\right), \\
& \forall x=\left(x_{1}, \ldots, x_{n}\right) \in \mathbb{R}^{n} .
\end{aligned}
$$

By analogy, in copulas framework, there is no confusion between the suvival copula $\bar{C}$ of $X$ (the copula of the survival function of $X$ ) and the cocopula $\widetilde{C}$ of $X$ (the survival distribution of the distribution $C)$.

Then, for all $\left(u_{1}, \ldots, u_{n}\right) \in[0,1]^{n}$, it follows that

$$
\begin{aligned}
\widetilde{C}\left(u_{1}, \ldots, u_{n}\right) & =P\left(U_{1}>u_{1}, \ldots, U_{n}>u_{n}\right) \\
& =\bar{C}\left(1-u_{1}, \ldots, 1-u_{n}\right) .
\end{aligned}
$$

Particularly in analysis with Archimedean copulas, survival distributions are often used to characterize exchangeability or the radial symmetry which is a key tool among the possibilities in which sense random vectors are symmetric. Following Nelsen [3], the random vector $\left(X_{1}, \ldots, X_{n}\right)$ is said to be radially symmetric about $\left(a_{1}, \ldots, a_{n}\right) \in \mathbb{R}^{n}$ if its joint distribution function $F$ satisfies

$$
\begin{aligned}
F\left(x_{1}+a_{1}, \ldots, x_{n}+a_{n}\right)=\bar{F}\left(a_{1}-x_{1}, \ldots, a_{n}-x_{n}\right) \\
\forall\left(x_{1}, \ldots, x_{n}\right) \in \mathbb{R}^{n},
\end{aligned}
$$

where $\bar{F}$ is the survival function of $F$. In terms of copulas relation (15) means that

$$
\begin{aligned}
& C\left(u_{1}, \ldots, u_{n}\right)=\bar{C}\left(u_{1}, \ldots, u_{n}\right) \\
& \forall\left(u_{1}, \ldots, u_{n}\right) \in[0,1]^{n} .
\end{aligned}
$$

\section{Main Results}

3.1. Spatial Dependence of Marginal Archimax Process. Let $X_{s}=\left\{\left(X_{s, 1}, \ldots, X_{s, n}\right), s \in S\right\}$ be a continuous stochastic random vector observed at a finite number of locations $S_{m}=\left\{s_{1}\right.$, $\left.\ldots, s_{m}\right\}, s_{i} \in \mathbb{R}^{2}$, with joint distribution $F_{s}=\left(F_{s, 1}, \ldots, F_{s, n}\right)$ whose underlying parametric copula is $\left\{C_{s}, s \in S\right\}$.

For simplicity reasons, let us note $X_{s, i}=X_{i}^{\check{s}}$ (which is different from $X_{i}^{s}$, the $s$ th power of $X_{i}$ ).

Under this notational assumption, the process $X_{s}$ is given by $X_{s}=\left\{\left(X_{1}^{\check{s}}, \ldots, X_{n}^{\check{s}}\right), s \in S\right\}$ and $F_{s}=\left(F_{1}^{\check{s}}, \ldots, F_{n}^{\check{s}}\right)$. We extend the concept of Archimax copula as follows.
Definition 3. The process $\left\{X_{s}, s \in S\right\}$ is called 2-marginally Archimax if for all $i, j \in\{1, \ldots, n\}$ every bivariate marginal distribution $F_{i, j}^{\check{s}}=\left(F_{i}^{\check{s}} ; F_{j}^{\check{s}}\right)$ can be associated with an Archimax copula $C_{\phi_{s}, A}$ with a completely monotone function $\phi_{s}$.

Consider, for example, the process $\left\{X_{s}, s \in S\right\}$ whose underlying copula is the following nested model:

$$
\begin{aligned}
& C_{m, \phi}\left(u_{1}^{\check{s}}, \ldots, u_{m}^{\check{s}}\right) \\
& \quad=\phi\left[\phi^{-1}\left(u_{1}^{\check{s}}\right)+\phi^{-1}\left(C_{m-1, \phi}\left(u_{2}^{\check{s}}, \ldots, u_{m}^{\check{s}}\right)\right)\right]
\end{aligned}
$$

for all $\left(u_{1}^{\check{s}}, \ldots, u_{m}^{\check{s}}\right) \in[0,1]^{m}$, where $C_{m, \phi}$ is an $\phi$-Archimedean copula. For the simple case where $\phi_{\theta}\left(x_{t}\right)=\left(-\ln x_{t}\right)^{\theta}, \quad \theta \geq$ 1 , the bivariate marginal copulas are the models of Gumbel, such that, for all site $s \in S$ and for all $i, j \in\{1, \ldots, n\}$,

$$
C_{s, \theta}\left(u_{i}^{\check{s}} ; u_{j}^{\check{s}}\right)=\exp \left\{-\left[\left(-\ln u_{i}^{\check{s}}\right)^{\theta}+\left(-\ln u_{j}^{\check{s}}\right)^{\theta}\right]^{1 / \theta}\right\} \text {, }
$$

which belongs to the class of Archimax models for $A(s) \equiv$ $1, s \in[0,1]$ with $i, j \in\{1, . ., n\}$.

Consider a 2-marginally Archimax process $\left\{X_{s}, s \in S\right\}$ with joint distribution function $F_{s}$. Assume that $X_{s}$ satisfies the regularly varying property with index $\alpha(\alpha>0)$, written as $F_{s} \in \mathrm{RV}(\alpha)$. That means particularly that there exists a random vector $Y_{s}$, distributed on the unit spatialized parametric simplex,

$$
\Delta_{s, n}=\left\{x_{s} \in \mathbb{R}_{+}^{n} ;\left\|x_{s}\right\|=\sum_{i=1}^{n} x_{s, i}=1\right\}
$$

such that, for any $s>0$ and any Borel-set $B_{s} \subset \Delta_{s, n}$,

$$
\frac{P\left(\left\|X_{s}\right\|>t y_{s}, Y_{s} /\left\|Y_{s}\right\| \in B_{s}\right)}{P\left(\left\|Y_{s}\right\|>y_{s}\right)} \underset{y_{s} \rightarrow+\infty}{\stackrel{v}{\longrightarrow}} P\left(Y_{s} \in B_{s}\right) t^{-\alpha},
$$

where $v$ symbolizes vague convergence and $\|\cdot\|$ is an arbitrary norm of $\mathbb{R}^{n}$ (see Nelsen [3]).

The following result characterizes the bivariate marginal distributions of Archimax processes.

Proposition 4. Let $\left\{X_{s}, s \in S\right\}$ be a 2-marginally Archimax process, with joint distribution $F_{s}$, and generated by

$$
\phi_{s}(t)=\int_{0}^{+\infty} e^{-w} d F_{s, 1}(w) .
$$

If there exists $m>0$ such as $\phi_{s}(1-1 / x) \in R V(-m)$, then every bivariate marginal distribution $F_{i, j}^{\check{s}}$ of $F_{s}$, related to $(i, j)$ with $1 \leq i, j \leq s$, is given by

$$
\begin{gathered}
F_{i, j}^{\check{s}}\left(x_{i}^{\check{s}}, x_{j}^{\check{s}}\right)=\phi_{s}^{-1}\left[\left(\sum_{k=i, j} \phi_{s}\left(F_{k}^{\check{s}}\left(x_{k}^{\check{s}}\right)\right)\right)\right. \\
\left.\cdot A_{s, m}^{*}\left(\frac{\phi_{s}\left(F_{i}^{\check{s}}\left(x_{i}^{\check{s}}\right)\right)}{\sum_{k=i, j} \phi_{s}\left[F_{k}^{\check{s}}\left(x_{k}^{\check{s}}\right)\right]}\right)\right],
\end{gathered}
$$

where $A_{s, m}^{*}$ is a space-varying, convex appropriate function mapping $[0,1]$ to $[1 / 2,1]^{m-1}$. 
Proof. Suppose that the processes $\left\{X_{s}, s \in S\right\}$ have a common bivariate marginal distribution; that is, for any site $s \in S$,

$$
F_{i, j}^{\check{s}}=\left(F_{i}^{\check{s}}, F_{j}^{\check{s}}\right)=\left(F_{1}^{\check{s}}, F_{2}^{\check{s}}\right) \quad \text { with } i, j \in\{1, \ldots, n\}
$$

and let $C_{\phi_{s}, A}$ denote the Archimax copula associated with the common bivariate margins of $F_{s}$.

Then, it follows that, for all $i, j \in\{1, \ldots, n\}$,

$$
\begin{gathered}
C_{\phi_{s}, A}\left(u_{i}^{\check{s}}, u_{j}^{\check{s}}\right)=\phi_{s}^{-1}\left[\left[\sum_{k=i, j} \phi_{s}\left(\left[F_{k}^{\check{s}}\right]^{-1}\left(u_{k}^{\check{s}}\right)\right)\right]\right. \\
\left.\cdot A\left(\frac{\phi_{s}\left(\left[F_{i}^{\check{s}}\right]^{-1}\left(u_{i}^{\check{s}}\right)\right)}{\sum_{k=i, j} \phi_{s}\left(\left[F_{k}^{\check{s}}\right]^{-1}\left(u_{k}^{\check{s}}\right)\right)}\right)\right]
\end{gathered}
$$

whenever $\phi_{s}$ is such that $\phi_{x}^{-1}(1-t / r) \in \mathrm{RV}(-\alpha)$ with $\phi_{x}^{-1}(t)=$ $\int_{t}^{+\infty}(1-t / r)^{m-1} d r$.

According to Sklar's theorem via relation (1), for all $1 \leq$ $i, j \leq n$, it comes that

$$
C_{\phi_{s}, A}\left(\left[F_{i}^{\check{s}}\right]\left(x_{i}\right),\left[F_{j}^{\check{s}}\right]\left(x_{j}\right)\right)=F_{i, j}^{\check{s}}\left(x_{i}^{\check{s}}, x_{j}^{\check{s}}\right) .
$$

Therefore, for all $\left(x_{i}, x_{j}\right) \in \mathbb{R}^{2}$,

$$
\begin{gathered}
F_{i, j}^{\check{s}}\left(x_{i}^{\check{s}}, x_{j}^{\check{s}}\right)=\phi_{s}^{-1}\left[\left[\sum_{k=i, j} \phi_{s}\left(F_{k}^{\check{s}}\left(x_{k}^{\check{s}}\right)\right)\right]\right. \\
\left.\cdot A\left(\frac{\phi_{s}\left(F_{i}^{\check{s}}\left(x_{i}\right)\right)}{\sum_{k=i, j} \phi_{s}\left(F_{k}^{\check{s}}\left(x_{k}^{\check{s}}\right)\right)}\right)\right] .
\end{gathered}
$$

Furthermore, while characterizing extensions of Archimax distributions, Genest et al. [15] showed that if an Archimax copula $C^{*}$ with Pickands dependence function $A^{*}$ belongs to the max-domain of attraction (MDA) of another given Archimax copula $C$, then there exists $\alpha \in[0,1]$, such that

$$
A^{*}(t)=\left[t^{1 / \alpha}+(1-t)^{1 / \alpha}\right]^{\alpha} A^{1 / \alpha}\left[\frac{t^{1 / \alpha}}{t^{1 / \alpha}+(1-t)^{1 / \alpha}}\right]
$$

Consequently, combining (1) and (5), it follows that, for all $\left(x_{i}, x_{j}\right) \in \mathbb{R}^{2}$,

$$
\begin{gathered}
C_{\phi_{s}, A}\left(F_{i}^{\check{s}}\left(x_{i}\right), F_{j}^{\check{s}}\left(x_{j}\right)\right)=\phi_{s}^{-1}\left[\left[\sum_{k=i, j} \phi_{s}\left(F_{k}^{\check{s}}\left(x_{k}^{\check{s}}\right)\right)\right]\right. \\
\left.\cdot A\left(\frac{\phi_{s}\left(F_{i}^{\check{s}}\left(x_{i}\right)\right)}{\sum_{k=i, j} \phi_{s}\left(F_{k}^{\check{s}}\left(x_{k}^{\check{s}}\right)\right)}\right)\right] .
\end{gathered}
$$

In particular, in a bivariate spatial context, it follows that, for all $i, j \in\{1, . ., n\}$,

$$
\begin{gathered}
C_{\phi_{s}, A}\left(F_{i}^{\check{s}}\left(x_{i}\right), F_{j}^{\check{s}}\left(x_{j}\right)\right)=\phi_{s}^{-1}\left[\left[\sum_{k=i, j} \phi_{s}\left(F_{k}^{\check{s}}\left(x_{k}^{\check{s}}\right)\right)\right]\right. \\
\left.\cdot A_{s, m}^{*}\left(\frac{\phi_{s}\left(F_{i}^{\check{s}}\left(x_{i}^{\check{s}}\right)\right)}{\sum_{k=i, j} \phi_{s}\left(F_{k}^{\check{s}}\left(x_{k}^{\check{s}}\right)\right)}\right)\right],
\end{gathered}
$$

$A^{*}$ is the Pickands dependence function to which the copula in its domain of attraction $C$ belongs.

Finaly, by combining (1) and (29), we have the following where $A^{*}$ is expressed in terms of $A_{s, m}^{*}$ :

$$
\begin{gathered}
F_{i, j}^{\check{s}}\left(x_{i}^{\check{s}}, x_{j}^{\check{s}}\right)=\phi_{s}^{-1}\left[\left[\sum_{k=i, j} \phi_{s}\left[F_{k}^{\check{s}}\left(x_{k}^{\check{s}}\right)\right]\right]\right. \\
\left.\cdot A_{s, m}^{*}\left(\frac{\phi_{s}\left(F_{i}^{\check{s}}\left(x_{i}^{\check{s}}\right)\right)}{\sum_{k=i, j} \phi_{s}\left[F_{k}^{\check{s}}\left(x_{k}^{\check{s}}\right)\right]}\right)\right] .
\end{gathered}
$$

Thus, we obtain (22) which is satisfied as asserted.

Definition 5. The function $A_{s, m}^{*}$ is called a spatial dependence Archimax function.

3.2. Spatial Upper Tail Dependence of Archimedean Copulas. One of the key properties of copulas is that they remain invariant under strictly increasing transformations of the marginal laws. Therefore, the tail dependence parameter becomes a pure property of the copula associated with the random vector, that is, independent of marginal laws. Then, from Durante and Sempi [1] the copula-based version of relation (9) is given by

$$
\lambda^{U, k}=\lim _{u \rightarrow 1^{-}}\left(\frac{\bar{C}_{k}[1-u, \ldots, 1-u]}{\bar{C}_{n-k}[1-u, \ldots, 1-u]}\right) \quad \forall u \in[0,1],
$$

where $\bar{C}$ is the survival copula of $C$. Then, in parametric context it follows this result.

Proposition 6. Let $\left\{C_{\phi_{s}}, s \in S\right\}$ be a spatial Archimedean copula with generator $\phi_{s}$. Then, for all $k, k<n$, the parametric generalization to $k$ of the upper TDC is given analytically by

$$
\begin{aligned}
& \lambda^{U, k}=1 \\
& \quad+\lim _{u_{s, i} \rightarrow 1}\left[\sum_{k=1}^{n} \frac{(-1)^{k}}{1-u_{s, k}}\left(\sum_{S_{k} \subset N} C_{\phi_{s}}\left(u_{s, i_{k}}\right) ; i_{k} \in S_{k}\right)\right],
\end{aligned}
$$

for specific elements $S_{k}$ of the set of nonempty subsets of $\{1,2, \ldots, n\}$.

Proof. Assume that the copula $C_{\phi_{s}}$ is a spatial Archimedean one, for a given $s \in S$. That means particularly that, for all $\left(u_{1}^{\check{s}}, \ldots, u_{n}^{\check{s}}\right) \in[0,1]^{n}$,

$$
C_{\phi_{s}}\left(u_{1}^{\check{s}}, \ldots, u_{n}^{\check{s}}\right)=\phi_{s}^{-1}\left[\phi_{s}\left(u_{1}^{\check{s}}\right)+\cdots+\phi_{s}\left(u_{n}^{\check{s}}\right)\right] .
$$


Moreover, noting that every copula is also a cumulative distribution function (with uniform margins), we let $\widetilde{C}_{\phi_{t}}$ be the survival distribution $\bar{C}_{\phi_{t}}$.

Then, using relation (14) in a space-varying context, it follows that

$$
\begin{aligned}
\widetilde{C}_{\phi_{s}} & \left(u_{1}^{\check{s}}, \ldots, u_{n}^{\check{s}}\right) \\
& =P\left(F_{1}^{\check{s}}\left(x_{1}^{\check{s}}\right)>u_{1}^{\check{s}}, \ldots, F_{n}^{\check{s}}\left(x_{n}^{\check{s}}\right)>u_{n}^{\check{s}}\right) \\
& =\bar{C}_{\phi_{s}}\left(1-u_{1}^{\check{s}}, \ldots, 1-u_{n}^{\check{s}}\right) .
\end{aligned}
$$

Furthermore, from Charpentier et al. (see in [9]), it comes that

$$
\begin{aligned}
& \widetilde{C}_{\phi_{s}}\left(u_{1}^{\check{s}}, \ldots, u_{n}^{\check{s}}\right)=\sum_{k=0}^{n}\left[(-1)^{k}\right. \\
& \left.\quad \cdot\left(\sum_{v\left(u_{1}^{\check{s}}, \ldots, u_{n}^{\check{s}}\right) \in Z_{s}(n-k, n, 1)} C_{\phi_{s}}\left(v_{1}, \ldots, v_{n}\right)\right)\right],
\end{aligned}
$$

where $Z_{s}(M, N, \varepsilon)$ denotes the set $\left\{v \in[0,1]^{n} ; v_{i} \in\left\{u_{s}\right.\right.$, $\left.\varepsilon\}, \quad \sum_{i=1}^{n} 1_{\{\varepsilon\}}\left(v_{i}\right)=M\right\}$.

Then, for $\left(u_{1}^{\check{s}}, \ldots, u_{n}^{\check{s}}\right) \in[0,1]^{n}$, we can rewrite more simply formula (24) such that

$$
\begin{aligned}
& \widetilde{C}_{\phi_{t}}\left(u_{1}^{\check{s}}, \ldots, u_{n}^{\check{s}}\right) \\
& \left.\quad=\sum_{k=0}^{n}\left[(-1)^{k}\left(\sum_{S_{k} \subset N} C_{\phi_{s}}\left(u_{i_{1}}^{\check{s}}, \ldots, u_{i_{n}}^{\check{s}}\right) ; i_{j} \in S_{k}\right)\right)\right],
\end{aligned}
$$

where $S_{k}=\left\{i_{1}, \ldots, i_{k}\right\}$ contains $k$ elements of the set of nonempty subsets of $N=\{1,2, \ldots, n\}$ with $u_{i_{j}}^{\check{s}}=1$ for all $i_{j} \notin S_{k}$.

Therefore, using simultaneously (35) and (36), it comes that, for all $\left(u_{i_{1}}^{\check{s}}, \ldots, u_{i_{n}}^{\check{s}}\right) \in[0,1]^{n}$,

$$
\begin{aligned}
& \bar{C}_{\phi_{t}}\left(u_{1}^{\check{s}}, \ldots, u_{n}^{\check{s}}\right) \\
& \quad=\sum_{k=0}^{n}\left[(-1)^{k}\left(\sum_{S_{k} \subset N} C_{\phi_{t}}\left(1-u_{i_{1}}^{\check{s}}, \ldots, 1-u_{i_{n}}^{\check{s}}\right)\right)\right],
\end{aligned}
$$

where $S_{k}$ is rather such as $u_{i_{j}}=0$ for all $i_{j} \notin S_{k}$.

Specifically, by replacing in (37) the Archimedean copula by its analytical form (33), it comes that

$$
\begin{aligned}
\bar{C}_{\phi_{s}}\left(u_{1}^{\check{s}}, \ldots, u_{n}^{\check{s}}\right) \\
\quad=\sum_{k=0}^{n}\left[(-1)^{k}\left(\sum_{s_{k} \subset N} \phi_{s}\left(\sum_{j=1}^{n} \phi_{s}^{-1}\left(1-u_{i_{j}}^{\check{s}}\right)\right)\right)\right] .
\end{aligned}
$$

Furthermore, using relation (17) in a parametric case, it follows that

$$
\begin{aligned}
& \lambda^{U, k}=\lim _{u_{t} \rightarrow 1} \frac{\bar{C}_{\phi_{s}}\left(1-u_{s}, \ldots, 1-u_{s}\right)}{1-u_{s}} \\
& =\lim _{u_{t} \rightarrow 1}\left\{\sum_{k=0}^{n}\left[\frac{(-1)^{k}}{1-u_{t}}\left(\sum_{S_{k} \subset N} \phi_{s}\left(\sum_{j=1}^{n} \phi_{s}^{-1}\left(u_{s, i_{j}}\right)\right)\right)\right]\right\} .
\end{aligned}
$$

Finally, using the analytical form of $\bar{C}_{\phi_{t}}$ in (31), we obtain (32) as asserted.

3.3. Spatial Lower Tail Dependence of Archimax Copulas. While modeling stochastic dependence Schmitz (see in [16]) established the consistency of the copulas associated with stochastic processes. Particularly, that means in a Archimedean class that the lower marginal copulas are still Archimedean copulas.

Similarly, the copula-based version of relations (10) is given by

$$
\lambda^{L, k}=\lim _{u \rightarrow 0}\left(\frac{C_{n}(u, \ldots, u)}{C_{n-k}(u, \ldots, u)}\right), \quad \forall u \in[0,1],
$$

where $C_{n-k}$ is the marginal copula underlying the marginal vector of $(n-k)$ components $\widetilde{X}_{s, n-k}$ such that $\widetilde{X}_{s, n-k}=$ $\left(X_{k+1}^{\check{s}}, \ldots, X_{n}^{\check{s}}\right)$. The following result generalizes the spacevarying lower TDC in a marginal Archimax field.

Proposition 7. Let $\phi_{s}$ be the generator of a 2-marginally Archimax process $\left\{X_{s}, s \in S\right\}$. Then, for all $s \in S$ and $k(k<n)$, the generalized lower TDC, $\lambda_{s}^{L, k}$, is given by the following:

(i)

$$
\lambda_{s}^{L, k} \leq \lim _{u \rightarrow 0^{+}} R\left(\phi_{s}\left(u_{s}\right)\right)
$$

where $R$ is a specific ratio in ]0,1[ if $C_{\phi_{s}}$ is strictly Archimedean.

(ii)

$$
\lambda_{s}^{L, k}=\lim _{u \rightarrow 0^{+}} \exp \left\{-D_{\phi_{s}}\left(u_{s}\right)\right\}
$$

for a specific conditional measure $D_{\phi_{s}}$ if $C_{\phi_{s}}$ is strictly extremal.

Proof. From formula (40) in a parametric and spatial context, it follows that

$$
\lambda_{s}^{L, k}=\lim _{u_{s} \rightarrow 0}\left(\frac{C_{\phi_{s}}\left(u_{s}, \ldots, u_{s}\right)}{\bar{C}_{\phi_{s}, n-k}\left(u_{s}, \ldots, u_{s}\right)}\right), \quad \forall u_{s} \in[0,1],
$$

where $C_{\phi_{s}}$ in Archimax copulas. In other terms, $C_{\phi_{s}}$ can either take a strictly Archimedean form or satisfy the max-stability property of extremal copulas. 
(i) Let $C_{\phi_{s}}$ be strictly Archimedean copulas and so satisfy formula (2); then it follows that

$$
\begin{aligned}
\lambda_{s}^{L, k} & =\lim _{u_{s} \rightarrow 0}\left[\frac{\phi_{s}^{-1}\left(\sum_{i=1}^{k} \phi_{s}\left(u_{s}\right)\right)}{\phi_{s}^{-1}\left(\sum_{i=k+1}^{n} \phi_{s}\left(u_{s}\right)\right)}\right] \\
& =\lim _{u_{s} \rightarrow 0}\left[\frac{\phi_{t}^{-1}\left(k \phi_{s}\left(u_{s}\right)\right)}{\phi_{s}^{-1}\left[(n-k) \phi_{s}\left(u_{s}\right)\right]}\right],
\end{aligned}
$$

when the function $\phi_{s}$ belongs to class (2).

Then, $\phi^{-1}$ is stricly increasing from $[0,+\infty]$ to $[0,1]$ and $\phi(0)=1$ and then $\lambda_{s}^{L, k}=R\left(\phi_{s}\left(u_{s}\right)\right)<1$ as asserted. (ii) Suppose that $C_{\phi_{s}}$ are rather extreme values copulas. Then the canonical representation of Pickands (see in [14]) shows that

$$
\begin{array}{r}
C\left(u_{1}, \ldots, u_{n}\right) \\
=\exp \left\{\sum_{i=1}^{n} \widetilde{u}_{s, i} A_{C}\left(\frac{\tilde{u}_{s, i}}{\sum_{i=1}^{n} \widetilde{u}_{s, i}}, \ldots, \frac{\tilde{u}_{s, n-1}}{\sum_{i=1}^{n} \widetilde{u}_{s, i}}\right)\right\} \\
\text { with } \widetilde{u}_{s, i}=\log u_{s, i},
\end{array}
$$

where $A_{C}$ is the Pickands function of $C$ defined on $S_{n}$ and subject to valid properties given in (Nelsen [3]).

Thus, formula (45) gives

$$
\lambda^{L, k}=\lim _{u_{s} \rightarrow 0}\left[\exp \left\{\sum_{i=1}^{k} \widetilde{u}_{s, i} A_{C}\left(\frac{\tilde{u}_{i}}{\sum_{i=1}^{k} \widetilde{u}_{s, i}}, \ldots, \frac{\widetilde{u}_{n-1}}{\sum_{i=1}^{k} \widetilde{u}_{s, i}}\right)-\sum_{i=k+1}^{n} \widetilde{u}_{s, i} A_{C_{n-k}}\left(\frac{\widetilde{u}_{k+1}}{\sum_{i=k+1}^{n} \widetilde{u}_{s, i}}, \ldots, \frac{\widetilde{u}_{n-1}}{\sum_{i=k+1}^{n} \widetilde{u}_{s, i}}\right)\right\}\right],
$$

where $A_{C_{n-h}}$ is instead the Pickands function of $C_{n-k}$.

Finally, it follows that a convex measure $D_{\phi_{s}}$ mapping $\left[(\mathbb{R} \cup\{ \pm \infty\})^{n}\right]^{2}$ to $[0,1]$ is as follows:

$$
\begin{aligned}
D_{\phi_{s}}\left(u_{s}\right) & \\
= & \sum_{i=1}^{k} \widetilde{u}_{s, i} A_{C}\left(\frac{\widetilde{u}_{i}}{\sum_{i=1}^{k} \widetilde{u}_{s, i}}, \ldots, \frac{\widetilde{u}_{n-1}}{\sum_{i=1}^{k} \widetilde{u}_{s, i}}\right) \\
& -\sum_{i=k+1}^{n} \widetilde{u}_{s, i} A_{C_{n-k}}\left(\frac{\widetilde{u}_{k+1}}{\sum_{i=k+1}^{n} \widetilde{u}_{s, i}}, \ldots, \frac{\widetilde{u}_{n-1}}{\sum_{i=k+1}^{n} \widetilde{u}_{s, i}}\right) .
\end{aligned}
$$

Then $\lambda^{L, k}=\lim _{u_{s} \rightarrow 0} \exp \left\{-D_{\phi_{s}}\left(u_{s}\right)\right\}$ as asserted.

3.4. Stability of Survival and Spatialized Archimedean Copulas. Let $\left\{X_{s}, s \in S\right\}$ be a 2-marginally Archimax process with generator $\phi_{s}$. We obtain the following property.

Proposition 8. Let $\left\{\phi_{s} ; s \in S\right\}$ be the generator of a 2marginally Archimax process $X_{s}=\left\{\left(X_{1, s} ; \ldots ; X_{n, s}\right), s \in S\right\}$ with copulas $\left\{C_{i, \phi_{s}} ; s \in S ; 1 \leq i \leq n\right\}$. Let moreover $\left\{\bar{C}_{i, \phi_{s}} ; s \in\right.$ $S ; 1 \leq i \leq n\}$ denote the survival spatial copulas of the copulas $C_{i, \phi_{s}}$ of the process. Then, under the strictly extremal assumption of the copulas $\left\{C_{i, \phi_{s}}\right\}$ and for all $\alpha=\left(\alpha_{1}, \ldots, \alpha_{m}\right) \in \Delta_{m}$, the geometric combination

$$
\begin{aligned}
C_{\phi_{s}, \alpha}\left(u_{s} ; v_{s}\right)= & \bar{C}_{1, \phi_{s}}^{\alpha_{1}}\left(u_{s} ; v_{s}\right) \times \bar{C}_{2, \phi_{s}}^{\alpha_{2}}\left(u_{s} ; v_{s}\right) \times \cdots \\
& \times \bar{C}_{m-1, \phi_{s}}^{\alpha_{m-1}}\left(u_{s} ; v_{s}\right) \times \bar{C}_{m, \phi_{s}}^{\alpha_{m}}\left(u_{s} ; v_{s}\right)
\end{aligned}
$$

provides a space-parameter bivariate extremal copula.

Proof. Proof of Proposition 8 consists in establishing first that, for every $\alpha$, the copula $C_{\phi_{s}, \alpha}$ is also an extreme value copula. Following Diakarya [12] or Liebscher [17], one can use transformations $g_{j i}$ for $j \in\{1, \ldots, m\}$ and $i \in\{1, \ldots, n\}$, mapping $[0,1]$ to $[0,1]$ such as $g_{j i}(s)=s^{\alpha_{j}}$ to show that, in $n$-dimensional fields for all $\alpha=\left(\alpha_{1}, \ldots, \alpha_{m}\right) \in S_{m}$ and for all $\left(u_{1}, \ldots, u_{n}\right) \in[0,1]^{n}$, the geometric transformation

$$
\begin{array}{r}
\prod_{j=1}^{m} C_{j}\left(g_{j, 1}\left(u_{1}^{\check{s}}\right), \ldots, g_{j, n}\left(u_{n}^{\check{s}}\right)\right) \\
\quad=\prod_{j=1}^{m} C_{j}\left(\left(u_{1}^{\check{s}}\right)^{\alpha_{1}}, \ldots,\left(u_{n}^{\check{s}}\right)^{\alpha_{n}}\right)
\end{array}
$$

which gives

$$
\begin{aligned}
\prod_{j=1}^{m} C_{j} & \left(g_{j, 1}\left(u_{1}^{\check{s}}\right), \ldots, g_{j, n}\left(u_{n}^{\check{s}}\right)\right) \\
= & C_{1}\left(\left(u_{1}^{\check{s}}\right)^{\alpha_{1}}, \ldots,\left(u_{n}^{\check{s}}\right)^{\alpha_{n}}\right) \times \cdots \\
& \times C_{m}\left(\left(u_{1}^{\check{s}}\right)^{\alpha_{1}}, \ldots,\left(u_{n}^{\check{s}}\right)^{\alpha_{n}}\right)
\end{aligned}
$$

is still a copula for any family like in time series of $n$ dimensional copulas $C_{1}, \ldots, C_{m}$ radially symmetric.

Therefore, in a bivariate and space-varying framework, the following transformation is still a copula:

$$
\begin{aligned}
C_{\phi_{s}, \alpha}\left(u_{s} ; v_{s}\right)= & C_{1, \phi_{s}}\left(u_{s}^{\alpha_{1}} ; v_{s}^{\alpha_{1}}\right) \times C_{2, \phi_{s}}\left(u_{s}^{\alpha_{2}} ; v_{s}^{\alpha_{2}}\right) \times \cdots \\
& \times C_{m-1, \phi_{s}}^{\alpha_{m-1}}\left(u_{s}^{\alpha_{m-1}} ; v_{s}^{\alpha_{m-1}}\right) \\
& \times C_{m, \phi_{t}}\left(u_{s}^{\alpha_{m}} ; v_{s}^{\alpha_{m}}\right) .
\end{aligned}
$$

Moreover, any copula $C_{i, \phi_{s}}$ is extremal one by assumption and therefore verifies particularly the copula-based max-stability property given in bivariate parametric case as

$$
\begin{aligned}
& C_{j, \phi_{s}}^{k}\left(u_{s} ; v_{s}\right)=C_{j, \phi_{s}}\left(u_{s}^{k} ; v_{s}^{k}\right) \\
& \forall\left(u_{s}, v_{s}\right) \in[0,1]^{2}, k>0 .
\end{aligned}
$$


Then, using relation (52), it follows, for all $\left(\alpha_{1}, \ldots, \alpha_{m}\right) \in \Delta_{m}$, that

$$
\begin{aligned}
C_{\phi_{s}, \alpha}\left(u_{s} ; v_{s}\right)= & C_{1, \phi_{s}}^{\alpha_{1}}\left(u_{s} ; v_{s}\right) \times C_{2, \phi_{s}}^{\alpha_{2}}\left(u_{s} ; v_{s}\right) \times \cdots \\
& \times C_{m-1, \phi_{s}}^{\alpha_{m-1}}\left(u_{s} ; v_{s}\right) \times C_{m, \phi_{s}}^{\alpha_{m}}\left(u_{s} ; v_{s}\right) .
\end{aligned}
$$

Furthermore, using the radial symmetry of the copulas $\left\{C_{i, \phi_{s}}\right.$ avec $\left.1 \leq i \leq n\right\}$ we derive from (52) that

$$
\begin{aligned}
C_{\phi_{s}, \alpha}\left(u_{s} ; v_{s}\right)= & \bar{C}_{1, \phi_{s}}^{\alpha_{1}}\left(u_{s} ; v_{s}\right) \times \bar{C}_{2, \phi_{s}}^{\alpha_{2}}\left(u_{s} ; v_{s}\right) \times \cdots \\
& \times \bar{C}_{m-1, \phi_{s}}^{\alpha_{m-1}}\left(u_{s} ; v_{s}\right) \times \bar{C}_{m, \phi_{s}}^{\alpha_{m}}\left(u_{s} ; v_{s}\right) .
\end{aligned}
$$

A sufficient condition is to prove that $C_{\phi_{s}, \alpha}$ verifies the copula-based max-stability property, for all $k>0$,

$$
\begin{aligned}
C_{\phi_{s}, \alpha}\left(u_{s} ; v_{s}\right)= & \bar{C}_{1, \phi_{s}}^{k \alpha_{1}}\left(u_{s} ; v_{s}\right) \times \bar{C}_{2, \phi_{s}}^{k \alpha_{2}}\left(u_{s} ; v_{s}\right) \times \cdots \\
& \times \bar{C}_{m-1, \phi_{s}}^{k \alpha_{m-1}}\left(u_{s} ; v_{s}\right) \times \bar{C}_{m, \phi_{s}}^{k \alpha_{m}}\left(u_{s} ; v_{s}\right),
\end{aligned}
$$

which then gives, due to formula (53), for all $k>0$,

$$
\begin{aligned}
C_{\phi_{s}, \alpha}\left(u_{s} ; v_{s}\right)= & C_{1, \phi_{s}}^{k \alpha_{1}}\left(u_{s} ; v_{s}\right) \times C_{2, \phi_{s}}^{k \alpha_{2}}\left(u_{s} ; v_{s}\right) \times \cdots \\
& \times C_{m-1, \phi_{s}}^{k \alpha_{m-1}}\left(u_{s} ; v_{s}\right) \times C_{m, \phi_{s}}^{k \alpha_{m}}\left(u_{s} ; v_{s}\right) .
\end{aligned}
$$

Let us use again the fact that $C_{i, \phi_{s}}$ are extreme values models.

So, for all $\left(u_{1}, \ldots, u_{n}\right) \in I^{n}$ and $k>0$,

$$
\begin{aligned}
C_{\phi_{s}, \alpha}\left(u_{s} ; v_{s}\right)= & C_{1, \phi_{s}}^{\alpha_{1}}\left(u_{s}^{k} ; u_{s}^{k}\right) \times C_{2, \phi_{s}}^{\alpha_{2}}\left(u_{s}^{k} ; u_{s}^{k}\right) \times \cdots \\
& \times C_{m-1, \phi_{s}}^{\alpha_{m-1}}\left(u_{s}^{k} ; u_{s}^{k}\right) \times C_{m, \phi_{s}}^{\alpha_{m}}\left(u_{s}^{k} ; u_{s}^{k}\right) .
\end{aligned}
$$

Using a second time (27) it follows that

$$
\begin{aligned}
C_{\phi_{s}, \alpha}\left(u_{s} ; v_{s}\right)= & \bar{C}_{1, \phi_{s}}^{\alpha_{1}}\left(u_{s}^{k} ; u_{s}^{k}\right) \times \bar{C}_{2, \phi_{s}}^{\alpha_{2}}\left(u_{s}^{k} ; u_{s}^{k}\right) \times \cdots \\
& \times \bar{C}_{m-1, \phi_{s}}^{\alpha_{m-1}}\left(u_{s}^{k} ; u_{s}^{k}\right) \times \bar{C}_{m, \phi_{s}}^{\alpha_{m}}\left(u_{s}^{k} ; u_{s}^{k}\right) .
\end{aligned}
$$

Hence $C_{\phi_{s}, \alpha}$ satisfies relation (52) which characterizes the extremal copulas.

\section{Conclusion and Discussion}

The results of this study provide important properties on parametric copulas and tail dependence in a spatial context. Properties have been proposed on spatial dependence for stochastic processes with bivariate marginal copulas in the Archimax class. More specifically, in a spatialized framework the characterization of the tail dependence concept has been extended to multivariate Archimedean copulas, for the upper coefficient, and to $n$-dimensional copulas with Archimax bivariate marginal, for the lower tail coefficient. Otherwise spatialized bivariate Archimedean copulas have been shown to be stable under geometric combinations.

These are very interesting results for a number of reasons. For example, the characterization of the tail dependence parameters provides an explicit form involving more computability. Moreover, these results allow us to extend stochastic processes analysis to marginal Archimax families. The particularity of our paper in stochastic processes analysis is that it investigates both survival and conditional properties of Archimax copulas in a parametric spatial and parametric context.

\section{Competing Interests}

The authors declare that there are no competing interests regarding the publication of this paper.

\section{References}

[1] F. Durante and C. Sempi, Principles of Copula Theory, Chapman \& Hall-CRC, Boca Raton, Fla, USA, 2016.

[2] H. Joe, Multivariate Models and Dependence Concepts, vol. 73 of Monographs on Statistics and Applied Probabilty, Chapman and Hall, London, UK, 1997.

[3] R. B. Nelsen, An Introduction to Copulas, vol. 139 of Springer Series in Statistics, Springer, New York, NY, USA, 2nd edition, 1999.

[4] A. Charpentier and J. Segers, "Tails of multivariate Archimedean copulas," Journal of Multivariate Analysis, vol. 100, no. 7, pp. 1521-1537, 2009.

[5] A. J. McNeil and J. Nešlehová, "Multivariate Archimedean copulas, $d$-monotone functions and $\ell_{1}$-norm symmetric distributions," The Annals of Statistics, vol. 37, no. 5, pp. 3059-3097, 2009.

[6] A. Di Ciaccio, M. Coli, and J. M. Angulo Ibanez, Eds., Advanced Statistical Methods for the Analysis of Large Data-Sets, Selected Papers of the Statistical Societies, Springer, Berlin, Germany, 2012.

[7] N. Whelan, "Sampling from Archimedean copulas," Quantitative Finance, vol. 4, no. 3, pp. 339-352, 2004.

[8] M. Hofert and M. Scherer, "CDO pricing with nested Archimedean copulas," Quantitative Finance, vol. 11, no. 5, pp. 775-787, 2011.

[9] A. Charpentier, A.-L. Fougères, C. Genest, and J. G. Nešlehová, "Multivariate archimax copulas," Journal of Multivariate Analysis, vol. 126, pp. 118-136, 2014.

[10] C. Genest and R. J. MacKay, "Copules archimédiennes et familles de lois bidimensionnelles dont les marges sont données," The Canadian Journal of Statistics, vol. 14, no. 2, pp. $145-159,1986$.

[11] EVANESCE Extreme value analysis employing statistical copula estimation, by René Carmona, http://www.orfe.princeton.edu/ $\sim$ rcarmona.

[12] B. Diakarya, "Analysis of stochastic spatial processes via copulas and measures of extremal dependence," Archives des Sciences, vol. 65, no. 12, pp. 665-673, 2012.

[13] F. Schmid, R. Schmidt, Th. Blumentritt, S. Gaier, and M. Ruppert, "Copula-based measures of multivariate association," in Copula Theory and Its Applications: Proceedings of the Workshop Held in Warsaw, 25-26 September 2009, vol. 198 of Lecture Notes in Statistics, pp. 209-236, Springer, Berlin, Germany, 2010.

[14] U. Cherubini, E. Luciano, and W. Vecchiato, Copula Methods in Finance, Wiley Finance Series, John Wiley \& Sons, Chichester, UK, 2004. 
[15] C. Genest, K. Ghoudi, and L.-P. Rivest, "A semiparametric estimation procedure of dependence parameters in multivariate families of distributions," Biometrika, vol. 82, no. 3, pp. 543-552, 1995.

[16] V. Schmitz, Copulas and stochastic processes [Ph.D. thesis], Aachen University, Aachen, Germany, 2003.

[17] E. Liebscher, "Modelling and estimation of multivariate copulas," Working Paper, University of Applied Sciences Merseburg, 2006. 


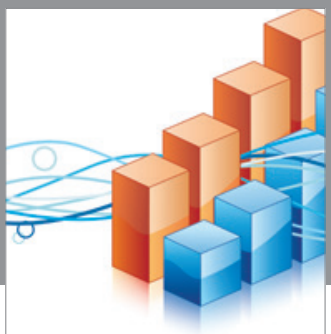

Advances in

Operations Research

vatem alat4

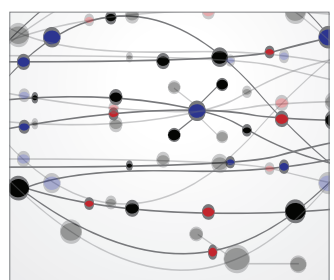

\section{The Scientific} World Journal
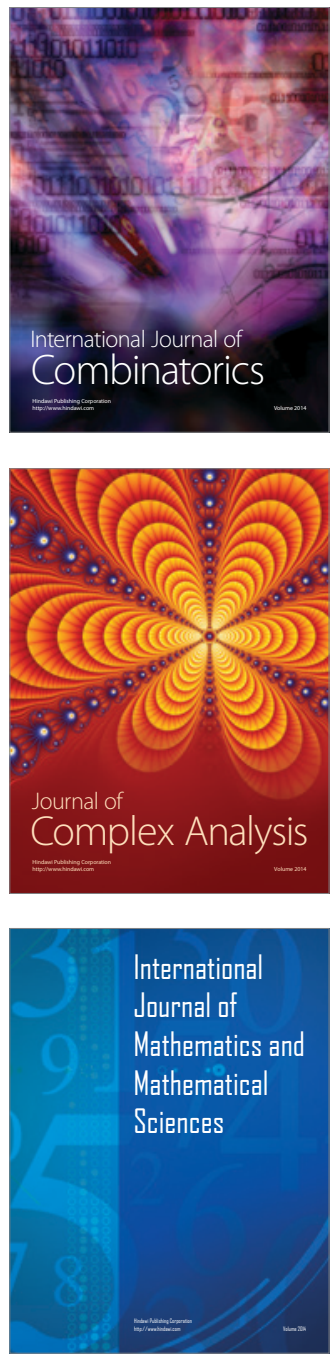
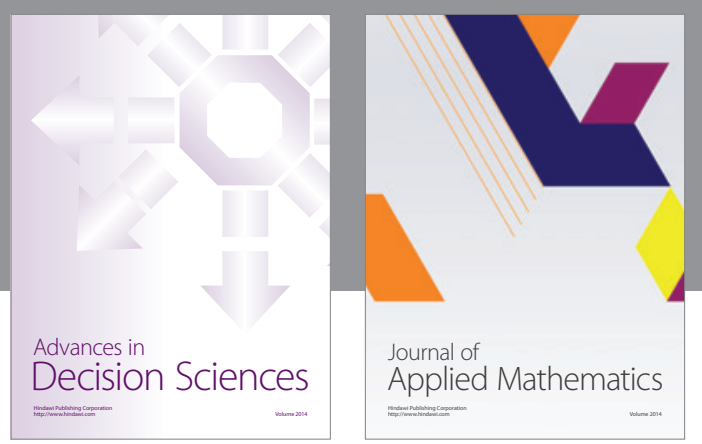

Algebra



\section{Hindawi}

Submit your manuscripts at

http://www.hindawi.com
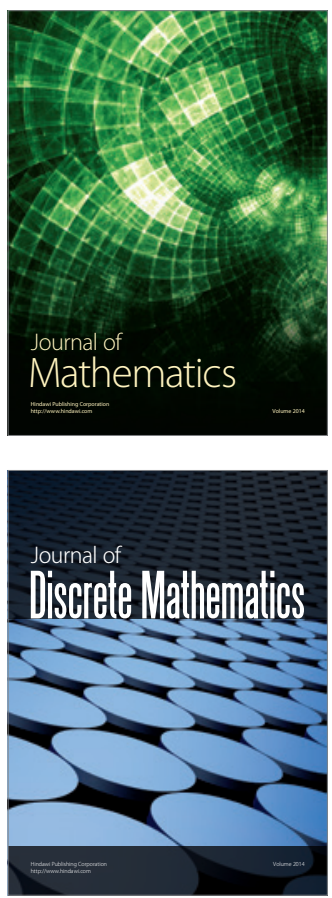

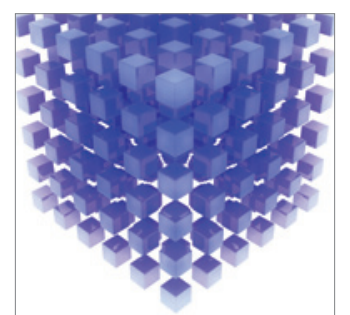

Mathematical Problems in Engineering
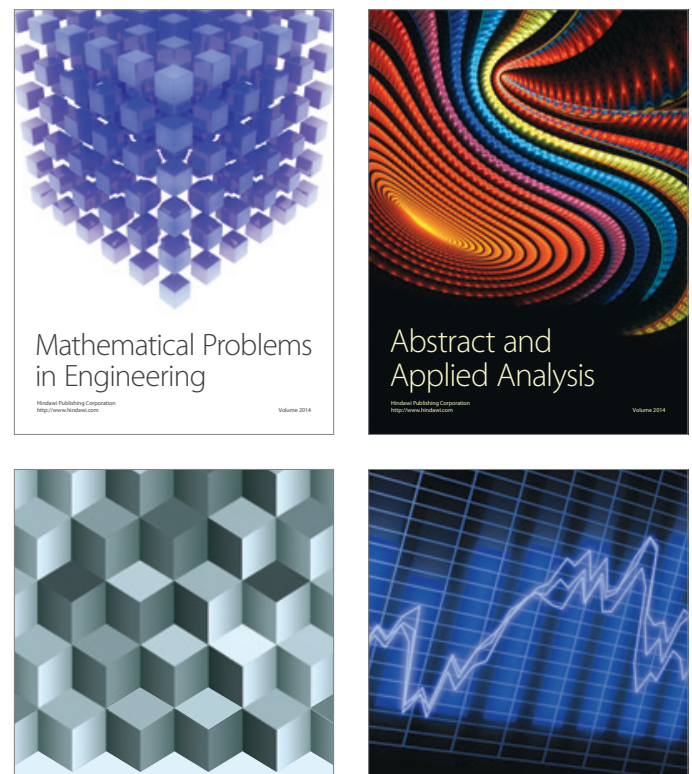

Journal of

Function Spaces

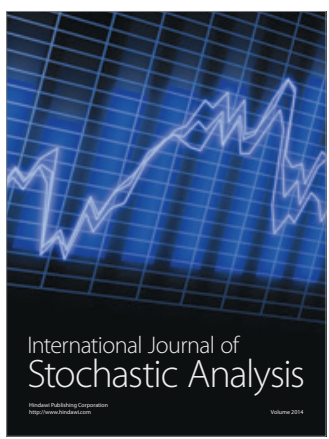

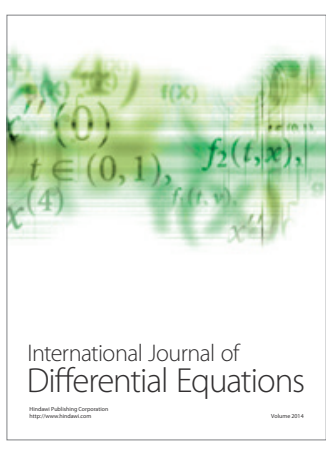
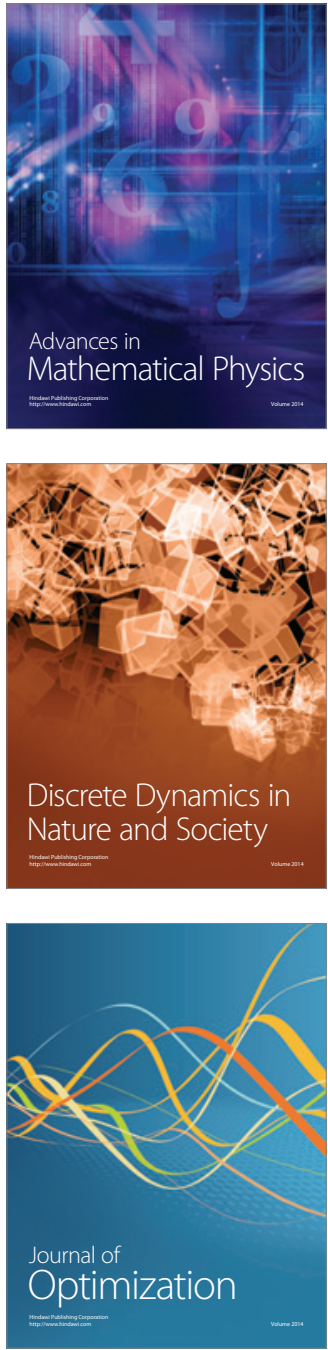